\title{
Some Results of Psycholinguistic Research of Polycode Advertising Texts: Gender Factor Influence
}

\author{
Antonina V. A nnenkoval,* \\ ${ }^{1}$ Southwest State University, Foreign Languages D epartment, 94, 50 Let Oktyabrya, 305040, Kursk, Russia
}

\begin{abstract}
Modern society is constantly changing and evolving, and the era of globalization and informatization leads to profound transformation of moral guidelines and personal space expansion due to deeper human immersion into the world of mass media. Image advertising is considered as one of the most common phenomena in the information era and can be characterized as a polycode text consisting of elements of different sign systems. The main purpose of such advertising is to change person's consumer behavior and form visions, desired by an advertising creator, in the consumer's individual lexicon. The article focuses on the study of peculiarities of image advertising visions functioning in the individual lexicon as well as their impact on an individual under a special advertising communicative situation depending on gender factor. To verify the thesis of differences in the strategies of image advertising perception applied by representatives of different gender groups, we have conducted the experimental research using the semantic differential; some results and their analysis are given in the article.
\end{abstract}

\section{Introduction}

Nowadays people are becoming more interested in advertising as a special means of communication due to its constant presence in all spheres of society and people's life. The development and change of the modern world lead to the integration of the mass media into cultural environment, making advertising an integral part of people's life, a certain cultural phenomenon, and language which reflects the state of society.

The specificity of image advertising visions functioning in the context of modern society is explained by the notion that advertising is considered as a special form of social communication where all information channels are used to transmit an assumed model of consumer behavior to any member of society. It should be noticed that such action influences the formation of "life standards" which are advantageous to an advertiser. Advertising is naturally incorporated into sociocultural processes which can be seen in any society. We believe that advertising is a special type of communicative situation in terms of which coded information is transmitted to change the addressee's way of thinking, his/her attitude towards a specific subject of advertising and a model of his/her consumer behavior.

Therefore, we have developed the classification of advertising based on the characteristics of a vision formation as a result of advertising impact on the individual. So, two types of advertising have been singled out. The first type is informational advertising which is considered as a text message, the main purpose of which is to give an addressee specific and informative content on an advertisement subject that will contribute to the subject further sales. The second type is image advertising which is understood as advertising aimed to image forming (positive or negative in dependence to goals set by an addressee). I mage is defined as a certain generalized vision formed in person's consciousness in response to impact of an advertising polycode text [1].

I mage advertising is a complex concept. $M$ anagement process of image advertising formation and operation in the mass media, society and image as well is subject to a vision symbolization process and occurs by conscious or subconscious matching of the ideal vision along with basic values acquired in a particular society with an individual's concepts and visions that he/she considers right for himself/herself personally. Thus, being a special sociocultural phenomenon, image advertising can be considered as some aspect of reality including external and internal object characteristics which are reflected in its image.

For the research use, image is defined as an idealized object emerging in person's individual lexicon. Whereas it is not possible to "measure" image directly, it can be estimated indirectly by means of the relationship emerged in communication, activities and a particular choice of a person. Event reality exists independently of a person. Therefore, image and image advertising, being a part of the mass culture which reflects all modern trends in the society development as well, naturally fit with the world, and change and shape new personal and social stereotypes and behaviors gradually.

Both Russian and foreign scientists are engaged in image and image advertising research [2-9]. But for all that, image advertising functioning as a complex, multidimensional social induced process remains not fully

* Corresponding author: Antonina-1984@yandex.ru 
understood. This statement defines the research relevance. To assess psycholinguistic factors influence on the "internal" visions formation in individual's mind while perceiving image advertising, we have conducted the experiment. Since the meaning of an advertising message is perceived only in terms of its symbolic and tangible content, the best method for achieving the goal, set in the advertising message, is to use symbolic visions and meanings that have already existed in person's mind.

During understanding of any text, including advertising one, an individual relies on his/her own schemes of world knowledge. This knowledge allows to see the situation that is described in the text, to think up it, to judge the believability or unreality of the events described. Therefore, we consider that it is necessary to identify psychophysiological characteristics of an individual, who perceives a text, separately.

Studying gender as one of the factors influencing the perception and formation of an imaginative component of image advertising will allow interpreting and analyzing the respondent's attitude towards a studied vision more fully and comprehensively, since gender is associated with linguistic, social, and psychophysiological characteristics of an individual. Gender factor has been studied in different branches of science from psychology and sociology to computer science and neurology [10-15].

Gender stereotypes of vision perception are determined by human environment, culture and society, so they determine the specificity of verbal and non-verbal human activity.

W e believe that the gender factor determines a specific set of behaviors emerged in most communicative situations, regardless of society nature or gender composition of people who are in communication with one another. Each person has his/her own specific strategies for information processing, a tendency to certain ways of knowledge categorizing. This affects the process of surrounding world perception. Thus, the result of the interaction of information processing strategies, which are formed under society and language influence, is the specific structures formation used for the perception process structuring. A forementioned structures occur in a network of associations, given to the proposed image advertising text, and in content of many incoming stimuli.

\section{Main Part}

\subsection{Methodology}

The experiment has been conducted to study the characteristics of gender influence on an advertising vision formation in the individual lexicon as well as on subjective assessments on the image advertising vision which is known or unknown to a respondent. The semantic differential method has been chosen as the main research method. It has allowed to study the degree of a vision perception of an image advertising without a verbal component.

It should be noted that the semantic differential is one of the cases of project study which gives possibility to take into account the fact that a stimulating situation makes sense not only because of its objective content, but also for reasons related to person's inclinations, in other words, due to respondent's subjectification and value which are attached to the current situation. Assessing stimuli according to given characteristics a respondent projects his/her properties into the situation [16]. In other words, the semantic differential is a useful tool for studying semantic spaces of a stimulus.

The semantic differential technique is widely used in Russian and foreign scientific literature to study respondents' perception of stimulus objects of a different nature such as verbal, visual, or polycode [8, 17-24]. One should not forget that the semantic differential can be used to study the level of formation and integrity of respondent's ideas about the world (cognitive diagnostics), as well as to study personal attitudes and emotional relationships towards given objects (projective techniques).

Person's attitude towards concepts that are significant to him/her in an advertising text can be found in his/her evaluative judgments, which are determined by logical dichotomies in person's consciousness (antonymous adjectives). Such bipolar evaluations are possible to be quantitatively measured by the semantic differential method [25].

Thus, the semantic differential is a technique that makes it possible to evaluate a value from both qualitative and quantitative sides. This fact has become a presupposition in choosing this particular method as the main one during the psycholinguistic experiment.

The hypothesis of the experimental study is the statement that the "gender" factor influences on the degree of a vision perception of an image advertising as well as on the formation of an image-medium vision in person's individual lexicon.

On the one hand an image advertising object reflects the reality, but on the other hand it distorts the reality through specific meanings focused on the vision of the object. As F. B artlett notes, knowledge comes out of the targeted process of remembrance [26]. Thus, knowledge activated by remembering sets up a firm relation between received information and a certain situation from the past which influences on perception of new stimulus, as the centre of an image advertising text is a vision which affects the effectiveness of influence on a respondent.

For our research we decided to choose 10 polycode texts of outdoor image advertising which are logos of industrial enterprises sorted by volume of production [27]. These advertising texts almost do not contain a verbal component which can be used for guessing or predicting company profile.

The participants of the experiment with the semantic differential method are 45 people belong to age group from 25 to 60 years old. A ll the respondents were divided into two groups by gender:

- the first group is the representatives of male in amount of 23 , the average age of participants is 30 ;

- the second group is the representatives of female in amount of 22 people, the average age of participants is 35 .

The total number of participants of the experimental study is 45 respondents. 
To carry out the psycholinguistic experiment we have chosen six factors suggested by V.F. Petrenko [28]. Each factor contains three bipolar adjective scales/pairs. Evaluation factor contains such adjective scales as pleasant-unpleasant, light-dark, and beautiful-ugly. The active-passive, excited-relaxed, and fast-slow adjective pairs define Activity factor. Order factor can be defined using scales regulated-chaotic, stable-changeable, and motionless-moving. Difficult-easy, secret-usual, and unlimited-limited scales are used to define Complexity factor. A djective pairs big-small, strong-weak, and heavylight define Potency factor. Comfortability factor has following scales safe-dangerous, soft-hard, and tenderrude.

The participants were to fill in a form of questionnaire. The respondents were to evaluate the objects in the table according to the given bipolar scales (from "-3" to "3") relying on their first impression. They should also put their gender, age and place of work or study on the form.

\subsection{Data Analysis Procedures}

45 completed forms from the participants have been obtained, these data have been analyzed and tabulated. We have done the vertical analysis to make exclusions of unw orkable data and it has not indicated any spoiled blank forms. It also should be noticed that respondents were interested in the experimental task fulfilling.

We have processed the obtained data by means of statistics methods and Microsoft Office Excel software.

The results of the research have been depicted in the graphic form of bar charts (See Fig. 1). The data are shown in averages of respondents' evaluations of image objects at 6 factors.

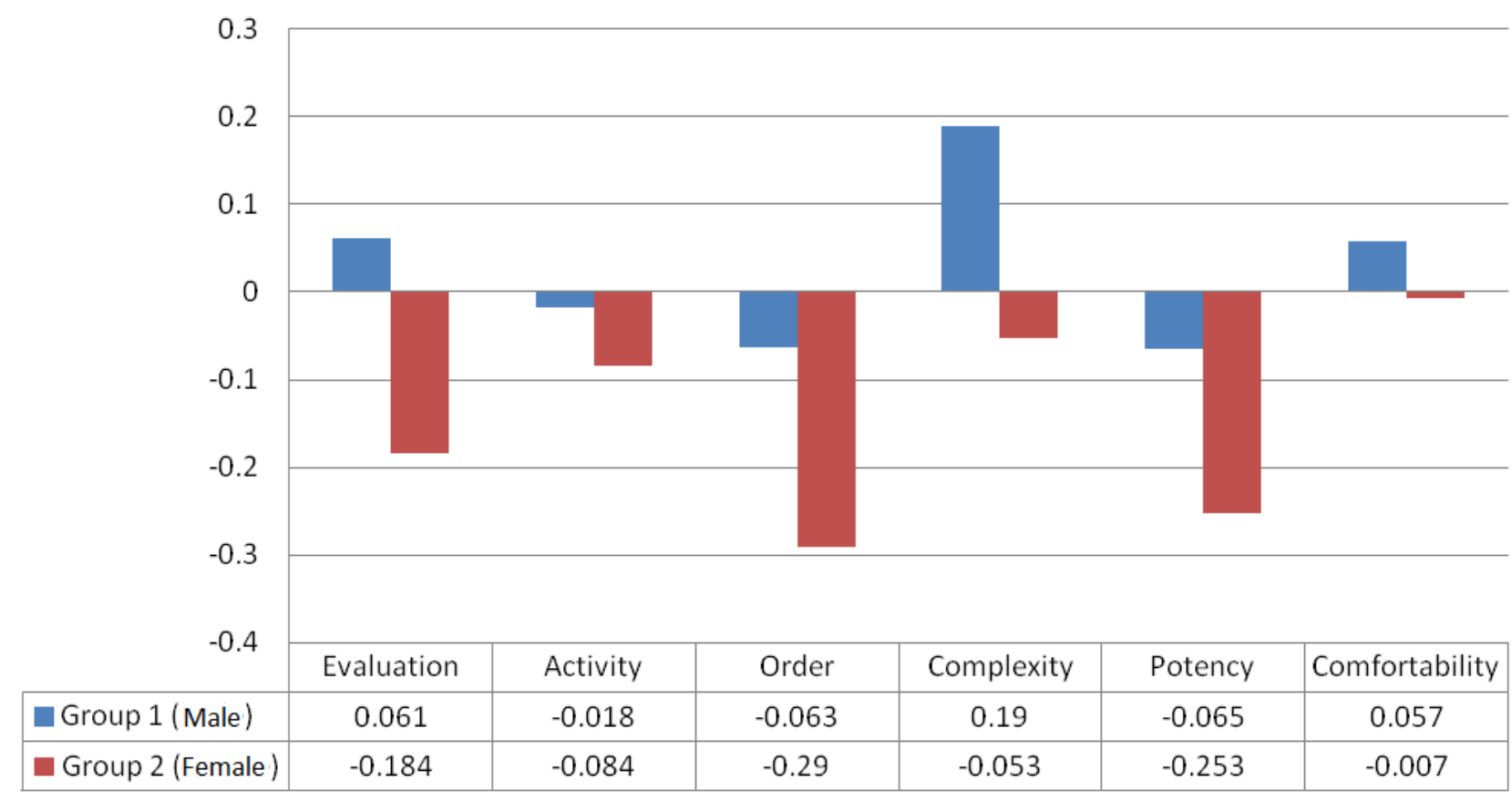

Fig. 1. Summarize data of the experiment

Judging by Figure 1 (see Fig. 1) we can see that the participants of the second gender group gave more clearly stated evaluations of advertising texts than the participants of the first gender group. Evaluation, Order and Potency factors are dominant factors in the second group, while Complexity factor is the only one dominant factor in the first group. A ctivity and Potency factors have the same orientation for both groups, while averages of the remained four factors are differently oriented. This means that figurative component is formed on the basis of the metaphorization process according to Evaluation, Order and Potency factors in female representatives and Complexity factor in male representatives.

The process of a vision identifying takes place in dependence on the context of individual's previous experience based on the most relevant features that can be determined using statistical methods, such as correlation analysis which consists in the determining of the degree of relationship between two random variables.
Correlation coefficients are used to find how strong relationship between experimental data is. The most common correlation coefficient, called the Pearson correlation coefficient, measures the strength of the linear association between variables. The range of values for the coefficient is -1.0 to 1.0. The greater the absolute value of a correlation coefficient, the stronger the linear relationship.

The literature shows common correlation relationship classification [29]: strong or tight relationship if coefficient of correlation (r) is $\geq 0.70$; medium if $0.50 \leq \mathrm{r}$ $\leq 0.69$; moderate if $0.30 \leq \mathrm{r} \leq 0.49$; weak if $0.20 \leq \mathrm{r} \leq$ 0.29 ; very weak if $r \leq 0.19$.

We have carried out correlation analysis for the experimental data. According to the results of the correlation analysis, almost all factors in the first group are in strong relationship with each other (e.g. Evaluation factor has high relationship with Activity, Complexity, Potency, and Comfortability factors, and Comfortability 
factor dependents on all other five factors). In the second group, on the contrary, there is high relationship only between Complexity and Evaluation factors, Complexity and Activity factors, and Comfortability and Evaluation factors. There is positive linear correlation between factors in both groups, which means that an increase in one variable (factor) is associated with an increase of another variable (factor). It should be noticed that in the second group we have found that Potency factor is al most independent from any other factor, and Comfortability factor has only very weak relationship with Order factor. We have not found similar cases in the first group.

In terms of mathematics a construction of a semantic space is a transition from large size basis sets given by scales (in this case there are 18 antonymous scales) to smaller-size basis sets given by factors (in this case there are 6 main factors). A ccording to V .F. Petrenko categories (factors) are rather rough meta-language which is used to describe stimulus objects. Consequently semantic spaces can be seen as a convenient way of values resolution into a fixed alphabet of categories (factors), i.e. semantic analysis of values can be carried out to make a decision about similarities and differences of the values within ndimensional semantic space [30].

It should be noted that the scales used in the experimental study are metaphorical expression of respondent's states and relations towards presented stimuli. This means that a respondent estimates image visions based not in the terms of these visions knowledge, but in the terms of his/her impressions that emerge when a person perceives a complex vision. For example, the vision of "Object 10" for respondents from the first gender group is ugly and hard, but for the respondents from the second group it is unpleasant, dark, difficult, limited, strong, hard, and rude, i.e. in this case the identity is occurred only on one scale of Comfortability factor. If to talk about image formed in respondent's individual lexicon, then characteristic scales of Potency and Order factors have the least influence on the figurative component representation and are minor components while implementing the cognitive mechanism of metaphorical transfer.

Considering the absence of a verbal component in an image-message which can be used to define an imagemedium, it should be noticed that respondents are usually failed while matching their background knowledge with the image-message, consequently respondents are to evaluate an image-medium vision by a non-verbal component as well as the vision formed in their individual lexicon. In the experimental research respondents stated unfamiliarity of presented stimuli regardless of the fact that chosen industrial enterprises are among the top leading enterprises in the region participants live in.

As the values of the objects within the semantic space are given by intersections of coordinate projections on axes of a semantic space they can be considered as an analogue of a categoric structure of an individual lexicon. According to V.F. Petrenko certain parameters of the semantic space correspond to certain aspects of cognitive organization of the individual lexicon [30].

Features of perception of an "O bject 5" vision and an "O bject 6" vision as well as construction of the visions in the individual lexicon can be evaluated considering graphic presentation of assessements given by representatives of different gender. Figure 2 (see Fig. 2) and Figure 3 (see Fig. 3) show "Object 5" and "Object 6" respectively and graphs of respondents' attitude towards image advertising texts for both gender groups.

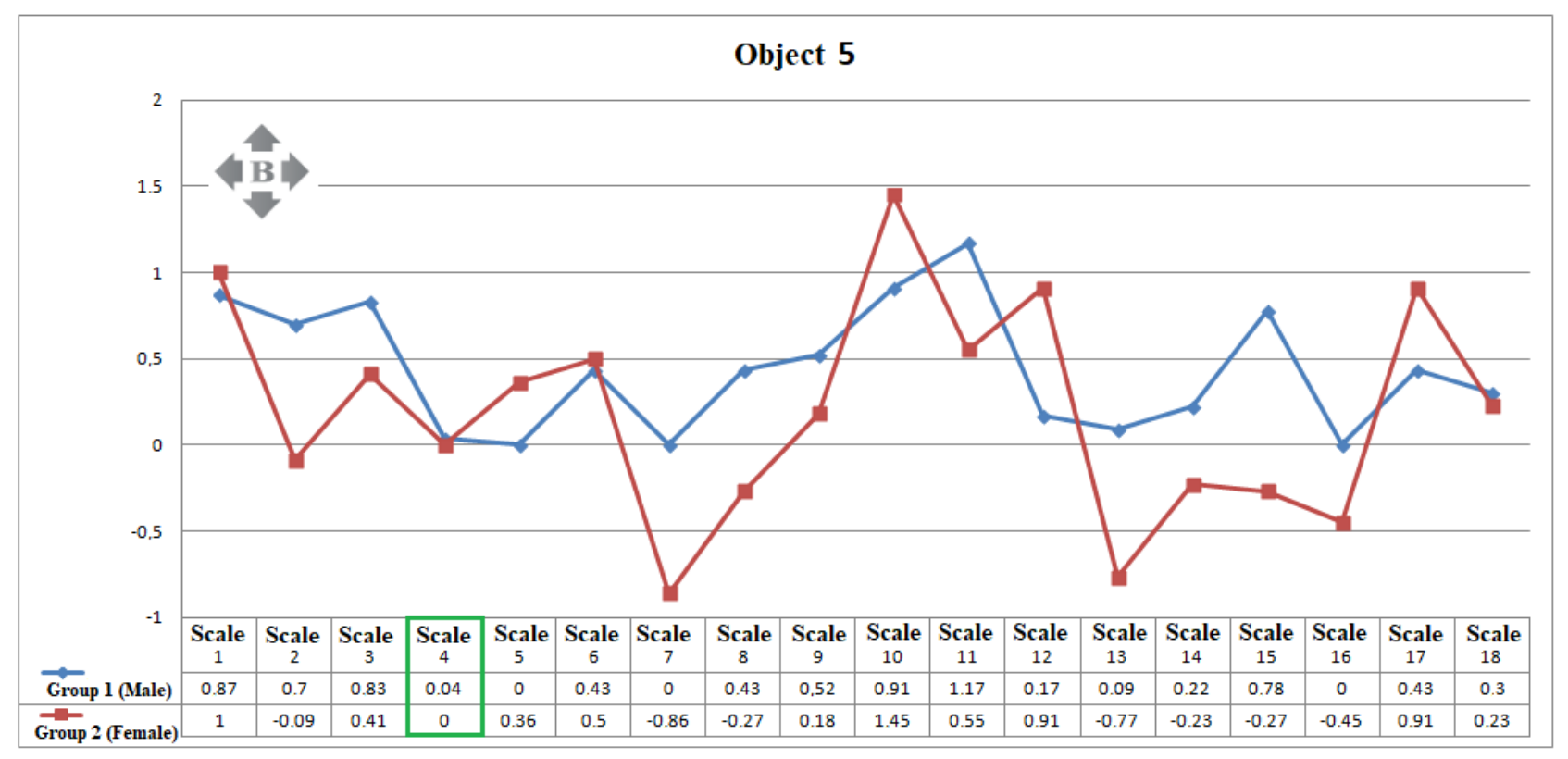

Fig. 2. Experiment results: Object 5 and graph of respondents' attitude towards Object 5 


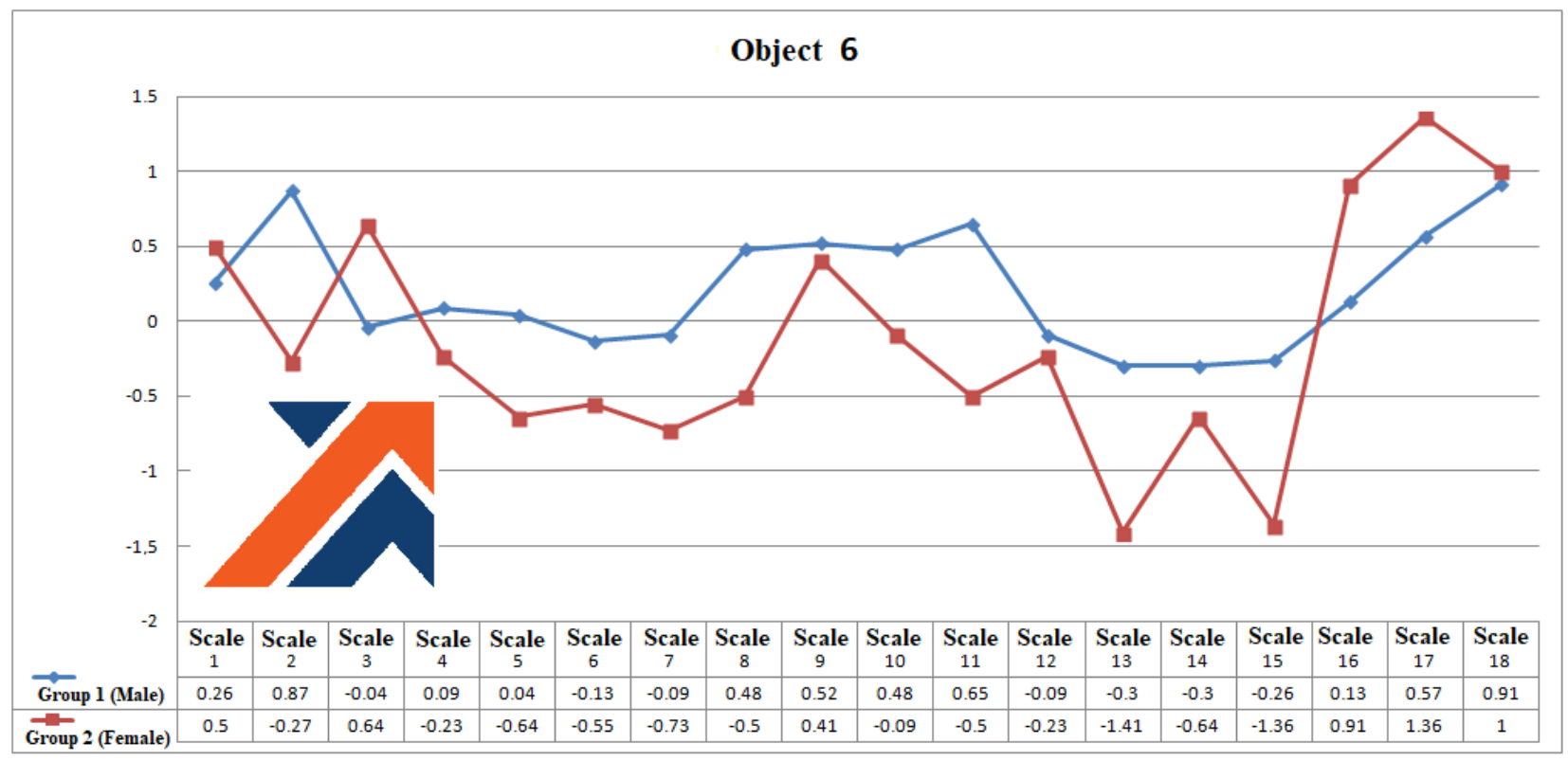

Fig. 3. Experiment results: Object 6 and graph of respondents' attitude towards Object 6

There is both verbal and non-verbal component in the image advertising text "Object 5" (see Fig. 2). The verbal component is represented only by the English letter ' $B$ ' which corresponds to the company name BIAXPLEN LTD (Kursk). The company is engaged in the production of polypropylene films and packages. The non-verbal component is represented by four gray arrows directed perpendicular to one another, forming a some kind of compass with the letter ' $B$ ' in the center instead of $a$ magnetic needle. This combination of the verbal and the non-verbal components based on the cognitive mechanism of metaphorical transfer creates a specific vision of the availability of goods produced by the company for any needs and requests.

There is only a non-verbal component in the advertising logo of "Object 6" (see Fig. 3). The picture shows an arrow composed of blue and orange lines and triangles. Such combination under the implementation of the cognitive mechanism of metaphorization creates a vision of steady movement upward to success and to constant development without any compromise.

According to the assessment given by the representatives of the first group (male) the vision of "Object 5" (see Fig. 2) is an unpleasant, dark, ugly, moving, easy, ordinary and rude, and the vision of "Object 6" (see Fig. 3) is dark, moving, hard and rude. As for assessment given by the representatives of the second group (female) the visions of the "Object 5" and "Object 6" can be described as unpleasant, regulated, easy, ordinary, limited, big and hard ("Object 5"), and as unpleasant, ugly, changeable, regulated, stable, secret, big, strong, heavy, dangerous, hard and rude ("Object 6") respectively.

Thus, these examples show that the representatives of the second group (female) have given approximately the same number or even more estimates to each stimulus object, and if the estimates given by the male representatives are based on the perceprion of a non- verbal part (easy, usual, dark, etc.), then the estimates by the female respondents are more "emotional" and include assessments of visions formed in the individual lexicon based on metaphorical transfer process.

If to check Figure 1 (see Fig. 1) once more as well as the given examples (see Fig. 2, Fig. 3) it can be obvious that the participants assess image advertising visions differently depending on their gender (as in the case of logos of industrial enterprises): visions formed in individual lexicons of the female representatives are multi-factorial and can be charactiresed by wide emotional spectrum; Complexity factor is dominat in the estimates given by the male representatives and the accentualization of the imaginative component is concrete and unemotional.

\section{Conclusion}

Image advertising vision carries information about an object, then this information is compared with person's background knowledge and experience and is the basis for the meanings formation when constructing a vision in person's individual lexicon. The perception of more "close" concepts and stimuli to a person is characterized by a greater frequency and figurativeness of given estimates, which clarifies the meaning of a vision in the recipien's individual lexicon and increases its detailing.

The characteristics compiling the implementation of the cognitive mechanism of metorization while the image advertising vision perception are directly depended on person's world image, personal experience, and background knowledge. We believe that a image advertising vision forms specific ideas about a subject and causes positive emotions that influence the addressee's behavior, while the vision formation takes into account the characteristics of the advertised object and the common features of the group of similar objects. According to the research data male and female gender 
stereotypes of the vision perception have been found out. These stereotypes are defined not only by biological characteristics, but also by the human environment, such as culture and society, so they determine features of verbal and non-verbal human activity.

Thus, basid on the analysis of the stimulus objects and graph of respondents' attitude towards these stimuli (see Fig. 2, Fig. 3), which describe the subjective perception of the stimulus objects visions by respondents of different gender groups, we can conclude the following: women react more emotionally than men. M ost averages given by female participants are higher than the same averages given by male respondents per practically every scale. At the same time female participantsCresponses approach toward extreme values of the bipolar antonymous scales. It should be emphasized that the subjective estimates given by the women in almost each studied feature scale are higher than the corresponding estimates given by the male participants.

The obtained data have proved the proposed hypothesis about the Gender factor influence on the perception of an imaginative component of image advertising: while decoding image-messages men and women construct an image-medium vision differently relying on different visions which are formed in respondents' individual lexicon under the implementation of the cognitive mechanism of metaphorization while percepioning image advertising visions.

Thus, the results of the experimental research show that gender of a society member affects the process of perception of the image advertising which is reflected in the subjective assessments given by individuals during the research using the semantic differential. We have found out that women react more emotionally. The process of formation of an internal vision that reflects their perception of the given image advertising text is characterized with more pronounced, more colorful assessment of an advertising image while male respondents showed the tendency to "smooth out" the quality of indicative scales resulting in lower scores which often tend to zero reflecting difficulties in rating an object to any particular quality.

The received data can be used for further study of the basic laws of influence of the gender factor on the formation of the certain individual's subjective reactions in response to the given stimuli as well as for identification of the specifics of the construction of the semantic space of the studied polycide texts using subjective assessments of different gender groups.

W e obtained the data it is possible to use for further study of the basic patterns of influence of gender factors and the formation of certain subjective reactions in an individual in response to the stimulus presented to him, as well as to identify the specifics of building the semantic space of the studied polycode advertising texts using subjective assessments of different gender groups.

\section{References}

1. O.S. Zubkova, A.V. Annenkova, Peculiarities of Image Advertising Visions Functioning in Individual
Lexicon: monograph (Southwest State University, A cademic B ook CJSC, K ursk, 2017)

2. R. Bell, A. Buchner, Positive Effects of Disruptive Advertising on Consumer Preferences, J ournal of Interactive $M$ arketing 41, 1-13 (2018)

3. M. Dehghani, M.K. Niaki, I. Ramezani, R. Sali, Evaluating the influence of YouTube advertising for attraction of young customers, Computers in Human B ehavior 59 (Jun), 165-172 (2016)

4. B. Dindaroglu, Competitive advertising on broadcasting channels and consumer welfare, Information Economics and Policy 42, 66-75 (2018)

5. A. Grunewald, M. Kräkel, Advertising as signal jamming, International Journal of Industrial Organization 55 (Nov), 91-113 (2017)

6. J. Huang, S. Su, L. Zhou, X i Liu, Attitude Toward the Viral Ad: Expanding Traditional Advertising Models to Interactive Advertising, Journal of Interactive M arketing 27(1) (Feb), 36-46 (2013)

7. K. Kim, S. Lee, Y.K. Choi, Image proximity in advertising appeals: Spatial distance and product types, J ournal of Business Research 99 (Jun), 490497 (2019)

8. O.S. Zubkova, A.V. Annenkova, Perception Peculiarities of Image Advertising Polycode Texts (Some Results of Experimental Research), M J LTM 7(8) (Aug), 189-202 (2017)

9. I. Petrovicia, Aspects of Symbolic Communications in Online Advertising, Procedia - Social and Behavioral Sciences 149, 719-723 (2014)

10. I. Borg, Age- and gender-related differences in the structure and the meaning of personal values, Personality and Individual Differences 138 (Feb), 336-343 (2019)

11. R. Grieve, E. March, G. Van Doorn, Masculinity might be more toxic than we think: The influence of gender roles on trait emotional manipulation, Personality and Individual Differences 138 (Feb), 157-162 (2019)

12. Y. Lee, W.-J.J. Y eung, Gender differences in correlates of cognition in first-episode psychosis, Social Science \& M edicine 229 (May), 87-95 (2019)

13. A. O ksuzyan, M.J. Dańko, J. Caputo, D. Jasilionisa, V.M. Shkolnikova, Is the story about sensitive women and stoical men true? Gender differences in health after adjustment for reporting behavior, Social Science \& M edicine 228 (May), 41-50 (2019)

14. M. Wong, J.C. Castro-Alonso, P. A yres, F. Paas, Investigating gender and spatial measurements in instructional animation research, Computers in H uman B ehavior 89 (Dec), 446-456 (2018)

15. N. Döring, A. Reif, S. Poschl, How genderstereotypical are selfies? A content analysis and comparison with magazine adverts, Computers in Human B ehavior 55(B) (Feb), 955-962 (2016)

16. V.A. Y adov, Sociological Research: Methodology, Programm, Methods (Nauka, M oscow, 1987) 
17. M. Chráska, M. Chrásková, Semantic Differential and its Risks in the Measurement of Students' Attitudes, Procedia - Social and B ehavioral Sciences 217, 820-829 (2016)

18. A. Ciabuca, The Development of a Semantic Differential Scale for Assessing the Perceived Image of Citizens about Romanian Police Forces, Procedia - Social and B ehavioral Sciences 187, 28-33 (2015)

19. Y u. Huang, Chun-Hsien Chen, Li Pheng Khoo, Products classification in emotional design using a basic-emotion based semantic differential method, International J ournal of Industrial Ergonomics 42(6), 569-580 (2012)

20. M. K lement, M. Chráska, M. Chrásková, The Use of the Semantic Differential Method in Identifying the Opininons Of University Students on Education Realized Through e-learning, Procedia - Social and B ehavioral Sciences 186, 1214-1223 (2015)

21. D.D. Palù, E. Buiatti, G.E. Puglisi, O. Houix, P. Susini, C. De Giorgi, A. A stolfi, The use of semantic differential scales in listening tests: A comparison between context and laboratory test conditions for the rolling sounds of office chairs, A pplied A coustics 127, 270-283 (2017)

22. A. Ploder, A. Eder, Semantic Differential, International Encyclopedia of the Social \& Behavioral Sciences (Second Edition), 563-571 (2015)
23. E.A. T anygina, The Study of Internal Image of Color Terms in Russian language Using the Semantic Differential Method, Herald of Vyatka State University 1-2, 35-40 (2011)

24. E .A. T anygina, The Image of Colour in the Linguistic Consciousness of the Native Speakers of Russian (the Russian Colour Term 'Pink'), Proceedings of I rkutsk State Linguistic U niversity 4, 69-75 (2011)

25. I.V. Zakharova, G.A. Stryukova, Semantic Differential as a Diagnostic Method of Teacher Perception by Students, Psychological Science and Education 3-4, 30-35 (1997)

26. F.C. B artl ett, Remembering: a Study in Experimental and Social Psychology (Cambridge U niversity Press, London, 1932)

27. Top 10 enterprises of Kursk (2015) URL: http://kursk.bezformata.ru/listnews/top-10-kurskihpredpriyatij/34035486/ (date of access: 06.05.2019)

28. V.F. Petrenko, Introduction to Experimental Psychosemantics: Study of the Forms of Representation in Everyday Consciousness (Publisher of M oscow University, Moscow, 1983)

29. A. Khomich, Correlation Analysis URL: http://khomich.narod.ru/metodichka/K orrelation/K or relation.htm (date of access: 06.05.2019)

30. V.F. Petrenko, Experimental psychosemantics: studies of individual consciousness, Questions of Psychology 5, 23-31 (1982). 